\title{
ON A FORMULA FOR THE $n$ TH DERIVATIVE AND ITS APPLICATIONS
}

\author{
VERA ČUlJAK AND Mirko S. Jovanović
}

Abstract. In this paper we obtain a formula which involves the $n$th derivative of the first order divided difference and a corresponding inequality for functions whose $(n+1)$-th derivative belongs to a $L^{p}$ space. These results are a generalization of the results from [1] and [2]. Finally, some examples are given.

Mathematics subject classification (2010): Primary 26D15, Secondary 26D99.

Keywords and phrases: Hölder inequality, $L^{p}$ space.

\section{REFERENCES}

[1] T. H. Gronwall, Problem 331., Amer. Math. Monthly, 20 (1913), 138.

[2] T. H. Gronwall, Problem 339., Amer. Math. Monthly, 20 (1913), 196.

[3] J. E. PeČarić, F. Proschan, And Y. L. Tong, Convex functions, partial orderings, and statistical applications, Academic Press, Inc, 1992. 